\title{
Ein Arzt auf literarischen Abwegen
}

\author{
D. Gaberell
}

Es gibt Menschen, die haben Lebensenergien, als hätte der Tag 30 Stunden, als müssten sie nie schlafen, als könnten sie unzählige Dinge unter einen Hut bringen - mühelos, gelassen und bescheiden. Sie sind eine Spezies rara, diese Menschen, Paul Wittwer ist zweifelsohne einer von ihnen.

Korrespondenz:

Daniel Gaberell

Mittelstrasse 19

Postfach 672

CH-3000 Bern 9

E-Mail: gaberell@herausgeber.ch
1959 geboren und als eines von fünf Kindern in Rüderswil im Emmental aufgewachsen, wechselte er in die Schulhäuser Zollbrück für die Sekundarschule, Burgdorf für das Gymnasium und später Bern für das Medizinstudium. Erst einen Monat vor Uni-Einschreibung entschied er sich für die Medizin. Er dachte eigentlich eher an Physik und Chemie, zu düster aber waren die Labor- und Kellerräume, zuwenig stand für ihn der Mensch im Vordergrund. Und als offene und spontane Person war er sich während der unterschiedlichen Assistenzzeiten auch immer sicher, dass er sich in jene Fachrichtungen spezialisieren wollte, in denen er gerade tätig war. Er arbeitete in den Spitälern Burgdorf, Insel und Tiefenau in Bern, und bei jedem Stellenwechsel organisierte er sich ein paar Monate Ferien. «Ich spürte immer, dass ich viel Zeit für mich und meine Familie brauche - ich musste mir schon immer Freiräume generieren», schildert Paul Wittwer. Und dies war dann auch mitunter ein Grund dafür, dass er sich gegen eine klassische Spitalkarriere entschied. Er wollte selbständiger Arzt werden mit einer eigenen Praxis, wo er sich ebendiese Freiräume einräumen und organisieren kann. «Zuerst dachte ich, es sei ein Kompromiss, die Wahl zum Allgemeinmediziner anstatt zum Spezialisten. Heute weiss ich die kreative Arbeit der vielen Krankheitsbilder und der unterschiedlichen Patienten zu schätzen», erklärt er. Seit 12 Jahren ist er einer von drei Hausärzten der Gemeinde Oberburg und führt eine Praxisgemeinschaft - was natürlich organisatorisch auch einige Vorteile mit sich bringt.

Trotzdem, 50 Stunden pro Woche ist er Arzt. Hat drei Kinder, lief vor ein paar Tagen den Jungfrau-Marathon und las soeben im Bücher Jäggi in Bern aus seinem ersten, knapp 400seitigen Kriminalroman. Wie schafft er all das?

«Ich schlafe sehr wenig», sagt er und lacht. Fünf bis sechs Stunden pro Nacht genügen ihm. An vielen Abenden sitzt er zu Hause, schaut zu

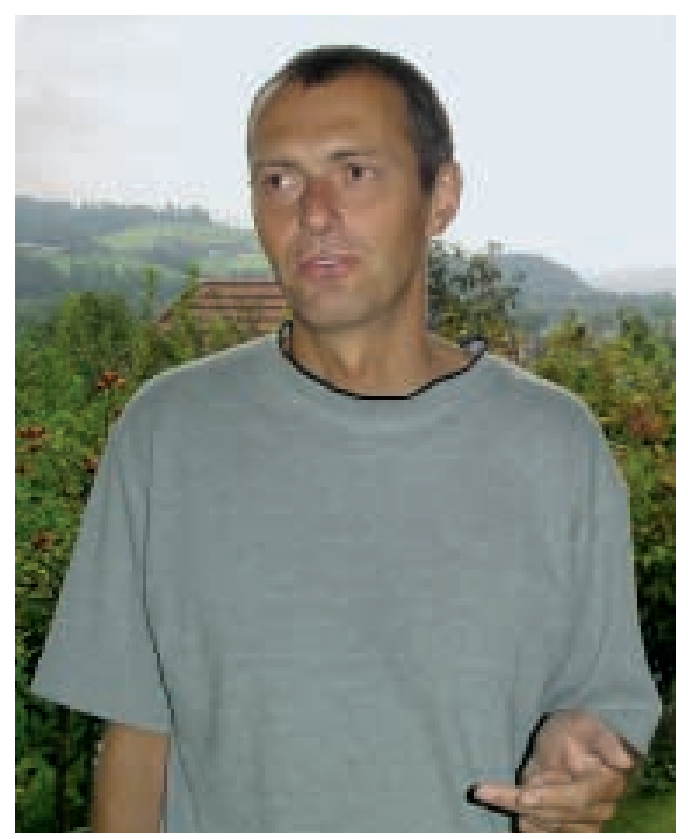

den Kindern oder hat ärztlichen Pikettdienst und während dieser nächtlichen Stunden schrieb er an seinem Roman. «Damals, 1998, in Südfrankreich unter einem schönen Feigenbaum, habe ich die ersten Worte niedergeschrieben», erinnert sich Wittwer. Er schrieb einfach, Wort für Wort, Satz um Satz, ohne zu wissen, wohin das führen wird. Die Geschichte kannte er schon lange in seinen Gedanken, aber ob er jemals das geplante Buch fertigschreiben würde und ob sich überhaupt ein Verlag für seinen Roman interessieren wird - all das wusste er nicht. Er schrieb sie fertig, seine Geschichte über die Missbräuche und Missstände der Medizin, und seine Frau als erste, ein befreundeter Arzt und ein Journalist später dienten als Kritiker. Und nach den üblichen Absagen von grossen Verlagen fand er mit Barbara Hirt vom Berner Nydegg-Verlag die ideale und engagierte Verlegerin für seinen Erstling.

Er hat auch Angst davor, dass die Kritiken allesamt schlecht ausfallen, dass sich das Buch nicht verkauft, dass an die Vernissage am Mittwoch keine Zuhörer kommen werden. Und ob er sich weiteren Romanprojekten widmen wird, hängt nicht zuletzt auch davon $\mathrm{ab}$, wie sein Buch bei den Lesern und Kritikern ankommen wird. 
«Ideen für eine neue Geschichte hätte ich schon, aber wenn es sich zeigt, dass mein Roman überall schlecht besprochen wird und die Verkaufszahlen unter den realistischen Erwartungen liegen, dann überlasse ich das Schreiben anderen.» Paul Wittwer weiss um seine Qualitäten, sowohl als Arzt wie als Autor. Einem Alex Capus könne er das Wasser nicht reichen, die Bilder von Capus, die er mit Worten generiert - so schreiben zu können, bewundert Wittwer sehr.
Er ist Arzt aus Überzeugung, und das will er auch bleiben. Sein Jüngster wird bald flügge, dann möchte er sich intensiver dem Segeln widmen und vielleicht zwei, drei Langstreckenläufe mehr absolvieren. Und natürlich hofft er auch auf eine Zukunft als Schriftsteller, aber das liegt nicht nur in seinen Händen.

Der Autor liest an den Burgdorfer Krimitagen am 4. November 2004 um 20 Uhr in der Stadtbibliothek Burgdorf.

\section{Kleine Büchermesse}

\section{Erhard Taverna}

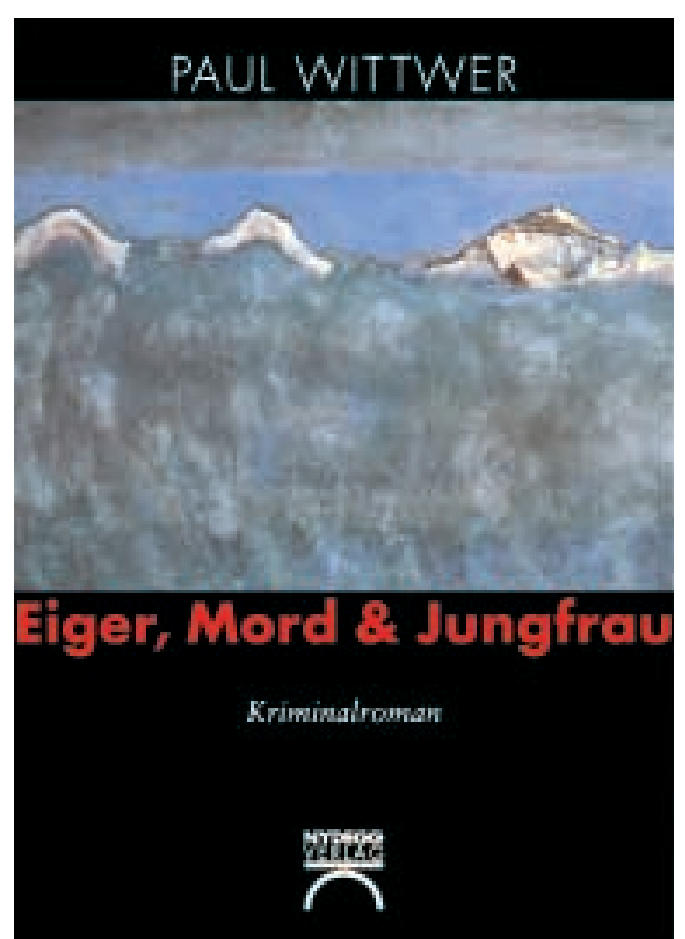

\section{Eiger, Mord \& Jungfrau}

Eine alte Freundschaft verpflichtet Franco zu Nachforschungen über den angeblichen Unfalltod seines Studienkollegen. Es kommen vor: das Inselspital Bern, die renommierte Privatklinik Eiger, an der die Herzchirurgen vertretungsweise mitwirken, ein undurchsichtiges Hilfswerk und ihr schwimmendes Lazarett im Mittelmeer. Weiter kommen vor die kaum öffentlich zugänglichen Labyrinthe des Kantonsspitals, gestresstes Personal auf den Notfallstationen, sich konkurrenzierende Ärzte, undurchsichtige Chefs und etliche zwielichtige Gestalten, die dem Helden ein Messer in die Brust und es auch sonst ziemlich bunt treiben. Ende gut und fast alles gut. Des Lesers langgehegte Vermutung wird bestätigt. Nach einem Show-down, der hier nicht verraten werden soll, entrinnt Franco knapp ein zweites Mal dem Sensenmann und darf dafür schwerverletzt seine geliebte Dimitra hinter dem Vorhang der Notfallkoje in die Arme nehmen.

Paul Wittwer, 1959 im Emmental geboren, Allgemeinmediziner in Oberburg bei Burgdorf, hat seinen ersten Krimi geschrieben. Einem etwas lang geratenen ersten Teil, in dem sich die Hauptfigur als Workaholic nur höchst widerwillig auf eine berufsfremde Recherche einlässt, folgt ein rasanter zweiter Teil mit allen Ingredienzien eines Thrillers. Wittwer gelingt dabei eine atmosphärisch dichte Schilderung eines Spitalbetriebes, den er als Assistenzarzt selber kennengelernt hat. Die Geschichte ist intelligent und solide konstruiert und trotz früh erkennbarer Zusammenhänge spannend erzählt. Gute Unterhaltung im Medizinermilieu mit Drehbuchqualitäten für den nächsten Schweizer Film.

- Paul Wittwer. Eiger, Mord \& Jungfrau. Kriminalroman. Bern: Nydegg Verlag; 2004. 396 Seiten. 


\section{Tasten auf dünnem Eis}

«Alles, was auf der Welt passiert, ist weniger schlimm, als zuviel gegessen zu haben. Keine Befriedigung so gründlich, wie vom Aufstehen bis zum Schlafengehen die Kontrolle behalten zu haben. Nichts berauschender als eine am Körper schlotternde Hose, die früher wie angegossen gesessen hat. Keine Frage wäre berückender als jene, ob Mara abgenommen hat, kein Kompliment süsser als die Feststellung, sie sei dünn geworden. Ausser, wenn Mara gerade Angstattacken hat, drehen sich ihre Gedanken ums Essen.» Mara liebt nur Tiere, das Klavierspielen hat sie aufgegeben, ihr Freund hat sie verlassen. Ihre Therapeutin überzeugt sie zu einem mehrwöchigen Klinikaufenthalt mit strikten Hausregeln, Einzelgesprächen und Gruppentherapie. Den Urlaub am Wochenende verbringt sie alleine mit der Katze, jeder Telefonkontakt bedeutet Qualen, der Einkauf von Esswaren ist ihr ein Greuel, jedes Kilogramm Gewichtszunahme macht angst und ist nur eine widerwillige Konzession an ihre Therapeuten. Die Geschichte wird auf drei zeitlichen Ebenen erzählt. In einer Gegenwart vom Klinikeintritt bis zum Ende, in einer Rückblende, die mit dem Entschluss zu diesem Klinikaufenthalt endet, und teilweise in ihrem Tagebuch. Karin Linsi, 1967 in Basel geboren, mit Lehrdiplom für Violoncello und Sprachdiplomen für Deutsch und Englisch, erzählt feinfühlig, ohne Selbstmitleid, ohne Schuldzuweisungen, ganz auf das Innenleben der Patientin konzentriert, die, «wenn schon einsam, dann einsam und mager» sein will. Es ist die traurige Geschichte einer Frau, die, vom täglichen Leben überfordert, nur noch in der Sucht einen Inhalt und Sicherheit findet. Und doch sind Begegnungen möglich, Gefühle wie Wut und Zärtlichkeit, Momente des Glücks und der Hoffnungen. Einfühlsam und ohne Illusionen ermöglicht die Autorin den Lesern einen Einblick in die stille Katastrophe von einer, «die gefangen ist in ihrer Welt, auf ihrem fernen Planeten, unter ihren Füssen nur dünnes Eis». Das Buch ist für alle Interessierten geschrieben, bestimmt kann es auch in der täglichen Praxisarbeit nützlich sein.

- Karin Linsi. Tasten auf dünnem Eis. Roman. Luzern; 2004. Herstellung und Verlag: Books on Demand GmbH, Norderstedt. 230 Seiten.

\section{Body Extensions}

$\mathrm{Zu}$ den menschlichen Obsessionen gehört der Wunsch, den Körper zu verändern. Die Publikation begleitet die Ausstellung «Body Extensions Wie wir den Körper erweitern», zu sehen kürzlich im Museum Bellerive in Zürich, neu in Lausanne, im «Musée de design et d'arts appliqués contemporains» vom 20. Oktober 2004 bis zum 30. Januar 2005. Die deutsch und französisch verfassten Beiträge sind der Kunst und Fotografie, dem Film und Comic und der Mode gewidmet, den reversiblen Eingriffen mit Mascara zur Verdoppelung des Wimpernvolumens oder dem gepolsterten Büstenhalter, bis zu den irreversiblen Einnahmen von Steroiden oder dem Selbstdesign durch operative Eingriffe. Blicke in die Archive volkskundlicher Museen verraten, dass die Spielarten der Körpererweiterungen die Proportionen seit je verfremdeten. Die Phantasie der Performancekünstler kennt keine Grenzen, noch weniger die der Modeschöpfer, Kulturen und Konventionen. Die Texte erklären und kommentieren. Sie vermitteln hervorragende Betrachtungen über die Kamera auf Bildern von Gustave Le Gray (1820-1884), phantastische Filmkörper wie Cyborgs und Superhelden wie Spiderman, Übertreibungen und Überzeichnungen in der Mode oder Konzeptmode versus Labels. Die vielen grossformatigen Bilder und Filme verblüffen, begeistern und schocken. Sie regen an und erregen, sie machen auch dankbar für die unerschöpfliche Kreativität, mit der der Homo sapiens seine Biologie überspielt.

Wer etwas bodennäher entschweben möchte, kann ergänzend die Sonderausstellung «bling bling» im Landesmuseum Zürich besuchen, wo ihn Traumstoffe aus St. Gallen in die Body Extensions der Haute Couture entführen. Eine ausgezeichnete Einführung dazu ist die Beilage zum Magazin «Hochparterre» Nr. 91/2004. Mehr unter www.bling-bling.ch.

- Claudia Pantellini, Peter Stohler (Hrsg.). Body Extensions. Stuttgart: Arnoldsche Verlagsanstalt; 2004. 192 Seiten, 123 Abbildungen in Farbe und schwarzweiss.

- Beilage zu «Hochparterre» 91/04, zu beziehen beim Schweizerischen Landesmuseum, Tel. 012186511. 